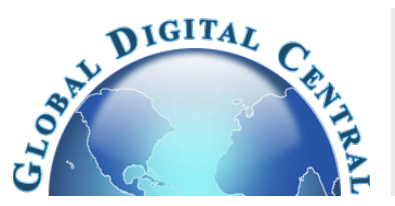

Frontiers in Heat and Mass Transfer

Available at www.ThermalFluidsCentral.org

\title{
GENERALIZED MAGNETO- THERMOELASTICITY AND HEAT CONDUCTION ON AN INFINITE MEDIUM WITH SPHERICAL CAVITY
}

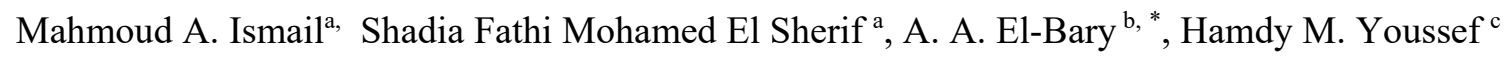 \\ ${ }^{a}$ Faculty of Science, Northern Border University, Arar, Kingdom of Saudi Arabia \\ ${ }^{b}$ Arab Academy for Science, Technology and Maritime Transport, P.O. Box 1029, Alexandria, Egypt \\ ${ }^{c}$ Mechanics Department, Faculty of Engineering and Islamic Architecture, \\ Umm Al-Qura University, Kingdom of Saudi Arabia
}

\begin{abstract}
In this paper we will discuss the problem of distribution of thermal stresses and temperature in a generalized magneto-thermo-viscoelastic solid spherical cavity of radius R according to Green- Naghdi (G-N II) and (G-N III) theory. The surface of the cavity is assumed to be free traction and subjected to a constant thermal shock. The Laplace transform technique is used to solve the problem. The state space approach is adopted for the solution of one dimensional problem. Solution of the problem in the physical domain are obtained by using a numerical method of MATLAP Programmer and the expression for the temperature, strain and stress are obtained. Numerical computations are carried out for a particular material for illustrating the results. Finally the results obtained are presented graphically to show the effect of time on the field.
\end{abstract}

Keywords: Thermal stress; Generalized Magneto-Thermo-Viscoelastic; Spherical Cavity; Green-Naghdi theory; Thermal shock.

\section{INTRODUCTION}

The theory of Thermo elasticity deals with the effect of mechanical and thermal disturbances on an elastic body. In the nineteenth century, Duhamel (1837) was the first to consider elastic problems with heat changes. In 1855 Neumann (1841) re derived the equations obtained by Duhamel using a different approach. Their theory, the theory of uncoupled thermo elasticity consists of the heat equation which is independent of mechanical effects and the equation of motion which contains the temperature as a known function. There are two defects of the theory. First, the fact that the mechanical state of the elastic body has no effect on the temperature. This is not in accord with true physical experiments. Second, the heat equation being parabolic, predicts an infinite speed of propagation for the temperature which again contradicts physical observations.

Biot (1956) introduced the coupled theory of thermo elasticity. In this theory the equations of elasticity and of heat conduction are coupled, which agree with physical experiments since any change of the temperature gives a certain amount of strain in an elastic body and vice versa. In most cases, the solutions which, are obtained by the classical theory, differ little from those obtained by using the theory of coupled thermoelectricity. This theory is useful in many problems. The equations of this theory consist of the equation of motion, which is hyperbolic partial differential equation, and of the equation of energy conservation, which is parabolic. There is a defect in this theory. The second equation being parabolic implies that if and elastic medium extending to infinity is subjected to a thermal or a mechanical disturbance, the effect will be felt instantaneously at infinitely distant points, which contradict experiments. Among the contributions in the subject of coupled thermo elasticity are the works of Nowacki who solved a problem for a half- space with heat sources (1957) and Ignaczak who solved a onedimensional problem for a spherical cavity (1959). Hetnarski solved a one-dimensional thermal shock problem (1961) and obtained the fundamental solution of the coupled theory (1964). Bahar and Hetnarski (1978) obtained the state space approach to the theory while Takeuti and Tsuji have solved a problem for a place due to rolling (1982). Uniqueness of the solution was proved by Weiner (1957) and some variation principles were obtained by Nickell and Sackman (1968).

Two theories of generalized thermo elasticity have been developed to oppose this last contradiction. One such generalization was obtained by Lord and Shulman (1976) and the other by Green and Lindsay (1972). In 1967 Lord and Shulman (1976) introduced the theory of generalized, thermo elasticity with one relaxation time for the special case of an isotropic body. This theory was extended by by Sherief (1980), Dhaliwal and Sherief (1980) in 1980 to include the anisotropic case. In this theory a modified law of heat conduction including both the heat flux and its time derivative replaces the conventional Fourier's law. The heat equation associated with this theory is hyperbolic and hence eliminates the paradox of infinite speeds of propagation inherent in both uncoupled and coupled theories of thermo elasticity.

Green and Lindsay (1972) developed the theory of generalized thermo elasticity with two relaxation times based on a generalized inequality of thermodynamics. This theory does not violate Fourier's law of heat conduction when the body under consideration has a center of symmetry. In this theory both the equations of motion and of heat conduction are hyperbolic. The equation of motion is modified and differs from that of the coupled thermo elasticity theory.

\footnotetext{
*Corresponding author.Email: aaelbary@aast.edu
} 
The study of viscoelastic behavior is of interest in several contexts. First, materials used in engineering applications may exhibit viscoelastic behavior as an unintentional side effect. Second, the mathematics underlying viscoelasticity theory within the applied mathematics community. Third, viscoelasticity is of interest in some branches of material science, metallurgy and solid-state-physics. Fourth, the causal links between viscoelasticity and microstructure is exploited in the use of viscoelastic tests as an inspection tools. In reality all materials deviate from Hooke's law in various ways, for example, by exhibiting viscous like as well as characteristics. Viscoelastic materials are those for which the relationship between stress and strain depends on time. All materials exhibit some viscoelastic response. In common metals such as steel, aluminum, copper etc. At room temperature and small strain, the behavior does not deviate much from linear elasticity. Synthetic polymer, wood as well as metals at high temperature display significant viscoelastic effects.

Modeling of viscoelastic response has a long tradition for describing a variety of phenomena (such as creep, relaxation and energy dissipation or damping) in structural analysis. One particular issue, which has been discussed extensively, is how complex. The linear viscoelastic model must be, i.e., what is the minimum number of material parameters that is required for an accurate description of the observed material behavior.

Most engineering materials exhibit a weak frequency dependence of the damping characteristics, which is difficult to describe with classic linear viscoelasticity that is based on integer rate laws for the pertinent internal variables. However, if the integer time derivative is replaced by a fractional order derivative operator, the number of parameters to describe damping in an accurate fashion can be significantly reduced. It has been argued, Bagley and Torvik (1983), the linear viscoelastic model together with fractional derivative have shown to be very flexible also for describing quasi static response, such as creep and stress relaxation. The Kelvin - Voigt model is one of the macroscopic mechanical models often used to describe the viscoelastic behavior of material. This model represents the delayed elastic response subjected to stress where the deformation is time dependent. Mishra et.al (1992) studied magnetothermo-mechanical interaction in an isotropic viscoelastic cylinder subjected to periodic loading considering Kelvin-Voigt model of linear viscoelasticity.

The theory of electro-magneto-thermo-viscoelasticity has aroused much interest in many industrial applications, particularly in nuclear devices, where there exists a primary magnetic field. Various investigations have been carried out by considering the interaction between magnetic, thermal and strain fields. Analyses of such problem also influence various applications in biomedical engineering as well as in different geomagnetic studied.

Due to the complicated nature of the governing equations for generalized electro-magneto- thermo viscoelasticity with thermal relaxation times, few attempts have been made to solve two-dimensional problems in this field. The propagation of electro magneto thermo elastic waves in an electrically and thermally conducting solid has been studied by many authors. Paria $(1962,1972)$ discussed the propagation of plane magneto-thermo elastic wave in an isotropic unbounded medium under the influence of a uniform thermal field and with magnetic field acting transversely to the direction of propagation, Paria used the classical Fourier's law of heat conduction and neglected the electric displacement together with charge density, the coupling between the current density and the heat flow density and the coupling between the temperature gradient and the electric current.

Result of important experiments determining the mechanical properties of viscoelastic material were involved by Koltunov's (1976). Bary et al. (2016) studied electro-thermoelasticity theory with memorydependent derivative heat transfer. Ezzat et al. (2015) linked the solution of two two-dimensional magneto-thermoelasticity with fractional order heat transfer in a medium of perfect conductivity. Youssef et al. (2014) obtained the solution of thermoelastic material response due to laser pulse heating in context of four theorems of thermoelasticity. Bary et al.
(2016) derived a new definition for linear Kelvin - Voigt model. Ismail (2014) analyzed state space approach for generalized thermoelastic medium subjected to non-uniform heat. Youssef (2014) presented Vibration of Gold Nano Beam in Context of Two-Temperature, Generalized Thermoelasticity Subjected to Laser Pulse. The solution of Two-temperature theory of magneto-thermo-viscoelasticity with fractional derivative and integral orders heat transfer, were obtained by Bary (2014). Ezzat et al. (2018) made great strides in the last decade in finding solutions for electro-magneto interaction in fractional GreenNaghdi thermoelastic solid with a cylindrical cavity. Many Applications of state space approach developed for different type of problems in thermoelasticity (2009-2018) some of them consider one temperature and other discussed two temperature.

\section{BASIC GOVERNING EQUATIONS}

We shall consider a homogeneous isotropic thermo-viscoelastic medium occupying the region $R \leq r<\infty$ of a perfect electrically conductivity permeated by an initial constant magnetic field $H_{o}$, where $R$ is the radius of the shell.

Due to the effect of this magnetic field there arises in the conducting medium an induced magnetic field $\boldsymbol{h}$ and induced electric field $\boldsymbol{E}$. Also, there arises a force $\boldsymbol{F}$ (the Lorentz Force). Due to the effect of this force, points of the medium undergo a displacement $u$, which gives rise to a temperature.

The linearized equations of electromagnetism for slowly moving media are:

curl $h=J+\varepsilon_{o} \frac{\partial E}{\partial t}$

$\operatorname{curl} E=-\mu_{o} \frac{\partial E}{\partial t}$

$B=\mu_{o} H$

$\operatorname{div} B=0$

The above field equations are supplemented by constitutive equations which consist first of ohm's law:

$E=-\mu_{o} \frac{\partial u}{\partial t} \times H_{o}$

The second constitutive equation is the one for the Lorenz force which is $F=J \times B$.

The third constitutive equation is the stress - displacement - temperature relation for viscoelastic medium of Kelvin - Voigt type:

$\sigma_{i j}=2 \mu_{e}\left(1+\alpha_{1} \frac{\partial}{\partial t}\right) e_{i j}+\lambda_{e}\left(1+\alpha_{o} \frac{\partial}{\partial t}\right) e \delta_{i j}-\gamma_{e}\left(1+\gamma_{o} \frac{\partial}{\partial t}\right) \theta \delta_{i j}$,

The equation of motion is given by:

$\rho \frac{\partial^{2} u_{i}}{\partial^{2} t}=\left(2 \mu_{e}\left(1+\alpha_{1} \frac{\partial}{\partial t}\right)+\lambda_{e}\left(1+\alpha_{o} \frac{\partial}{\partial t}\right)\right) u_{j, i j}-\gamma_{e}(1+$

$\left.\gamma_{o} \frac{\partial}{\partial t}\right) \theta_{, i}+\mu_{o}\left(J \times H_{o}\right)$

The generalized heat conduction equation is given by

$K \theta_{, i i}+K^{*}=\rho C_{E} \ddot{\theta}+\gamma_{e} T_{o}\left(1+\gamma_{o} \frac{\partial}{\partial t}\right) \ddot{e}$

The strain displacement relation is given by

$e_{i j}=\frac{1}{2}\left(u_{i, j}+u_{j, i}\right)$

Together with the previous equations, constitute a complete system of generalized -magneto-thermo-viscoelasticity equations for a medium with a perfect conductivity.

Let $(r, \psi, \phi)$ denote the radial coordinates, the co- latitude, and the longitude of a spherical coordinates system, respectively. Due to spherical symmetry, all the considered function will be functions of $r$ and $t$ only.

The components of the displacement vector will be taken the form:

$u_{r}=u(r, t), u_{\psi}=u_{\phi}=0$

The strain tensor components are thus given by

$e_{r r}=\frac{\partial u}{\partial r}, e_{\psi \psi}=e_{\phi \phi}=\frac{u}{r}, e_{r \phi}=e_{\phi \psi}=0$ 
It follow that the cubical dilatation is of the form

$e=\frac{\partial u}{\partial r}+\frac{2 u}{r}=\frac{1}{r^{2}} \frac{\partial\left(r^{2} u\right)}{\partial r}$

From equation (7) we obtain the components of the stress tensor as

$\sigma_{r r}=2 \mu_{e}\left(1+\alpha_{1} \frac{\partial}{\partial t}\right) \frac{\partial u}{\partial r}+\lambda_{e}\left(1+\alpha_{o} \frac{\partial}{\partial t}\right) e-\gamma_{e}\left(1+\gamma_{o} \frac{\partial}{\partial t}\right) \theta$

$$
\sigma_{\phi \phi}=\sigma_{\psi \psi}=2 \mu_{e}\left(1+\alpha_{1} \frac{\partial}{\partial t}\right) \frac{u}{r}+\lambda_{e}\left(1+\alpha_{o} \frac{\partial}{\partial t}\right) e-\gamma_{e}\left(1+\gamma_{o} \frac{\partial}{\partial t}\right) \theta
$$

$\sigma_{r \phi}=\sigma_{r \psi}=\sigma_{\psi \phi}=0$

Assume now that the initial magnetic field acts in the $\phi$-direction and has the components $\left(0,0, H_{o}\right)$.

The induced magnetic field $\mathrm{h}$ will have one component $\mathrm{h}$ in the $\phi-$ direction, while the induced electric field $\mathrm{E}$ will have one component $\mathrm{E}$ in the $\psi$-direction.

Then, equations (1), (2) and (5) yield

$J=H_{o} \frac{\partial e}{\partial r}$,

$h=-H_{o}\left(\frac{\partial u}{\partial r}+\frac{u}{r}\right)$,

$E=\mu_{o} H_{o} \frac{\partial u}{\partial t}$.

From equations (17) and (6), we get that the Lorentz force has only one component $F_{r}$ in the $r$-direction:

$F_{r}=\mu_{o} H_{o}^{2} \frac{\partial e}{\partial r}$

Also, we arrived at

$\rho \frac{\partial^{2} t}{\partial t^{2}}=\left(2 \mu_{e}\left(1+\alpha_{1} \frac{\partial}{\partial t}\right)+2 \lambda_{e}\left(1+\alpha_{o} \frac{\partial}{\partial t}\right)+\mu_{o} H_{o}^{2}\right) \frac{\partial e}{\partial r}-\gamma_{e}(1+$

$\left.\gamma_{o} \frac{\partial}{\partial t}\right) \frac{\partial e}{\partial r}$

Equation (21) is to be supplemented by the constitutive equation (13) and the heat conduction equation

$K \nabla^{2} \theta+K^{*} \nabla^{2} \dot{\theta}=\rho C_{E} \ddot{\theta}+\gamma_{e} T_{o}\left(1+\gamma_{o} \frac{\partial}{\partial t}\right) \ddot{e}$.

Where $\nabla^{2}$ is Laplace's operator in spherical coordinates which is given by

$\nabla^{2}=\frac{1}{r^{2}} \frac{\partial}{\partial r}\left(r^{2} \frac{\partial}{\partial r}\right)+\frac{1}{r^{2} \sin \psi}\left(\sin \psi \frac{\partial}{\partial \psi}\right)+\frac{1}{r^{2} \sin ^{2} \psi} \frac{\partial^{2}}{\partial \phi^{2}}$

In case of dependence on $r$ only, this reduce to

$\nabla^{2}=\frac{1}{r^{2}} \frac{\partial}{\partial r}\left(r^{2} \frac{\partial}{\partial r}\right)$

Now, we shall use the following non dimensional variables:

$\dot{r}=C_{1} \eta r, \dot{u}=C_{1} \eta u, \hat{t}=C_{1}^{2} \eta t, \gamma_{o}=C_{1}^{2} \eta \gamma_{o}, \alpha_{o}^{\prime}=C_{1}^{2} \eta \alpha_{o}$,

$\alpha_{1}^{\prime}=C_{1}^{2} \eta \alpha_{1}, \sigma_{l j}^{\prime}=\frac{\sigma_{i j}}{\mu_{e}}, \dot{\theta}=\frac{\theta}{T_{o}}, \hat{h}=\frac{h}{H_{o}}, \dot{E}=\frac{E}{\mu_{o} H_{o} C 1}, j=\frac{J}{\eta H_{o} C 1}$.

Equations (14) - (19), (21) and (22) take the following form (dropping the primes for convenience).

$$
\begin{aligned}
& J=\frac{\partial e}{\partial r} \\
& h=-\left(\frac{\partial u}{\partial r}+\frac{u}{r}\right) \\
& E=\frac{\partial u}{\partial t}, \\
& \sigma_{r r}=\frac{2 \mu_{e}}{\lambda_{e}+2 \mu_{e}}\left(1+\alpha_{1} \frac{\partial}{\partial t}\right) \frac{\partial u}{\partial r}+\frac{\lambda_{e}}{\lambda_{e}+2 \mu_{e}}\left(1+\alpha_{o} \frac{\partial}{\partial t}\right) e \frac{\gamma_{e} \theta_{o}}{\lambda_{e}+2 \mu_{e}}\left(1+\gamma_{o} \frac{\partial}{\partial t}\right) \theta \\
& \qquad \sigma_{\phi \phi}=\sigma_{\psi \psi}=\frac{2 \mu_{e}}{\lambda_{e}+2 \mu_{e}}\left(1+\alpha_{1} \frac{\partial}{\partial t}\right) \frac{u}{r}+\frac{\lambda_{e}}{\lambda_{e}+2 \mu_{e}}\left(1+\alpha_{o} \frac{\partial}{\partial t}\right) e- \\
& \frac{\gamma_{e} \theta_{o}}{\lambda_{e}+2 \mu_{e}}\left(1+\gamma_{o} \frac{\partial}{\partial t}\right) \theta
\end{aligned}
$$

$\sigma_{r \phi}=\sigma_{r \psi}=\sigma_{\phi \psi}=0$

$\frac{\partial^{2} u}{\partial t^{2}}=\left(1+\mu_{o} H_{o}^{2}+\frac{\lambda_{e} \alpha_{o}+2 \mu_{e} \alpha_{1}}{\rho C_{1}^{2}} \frac{\partial}{\partial t}\right) \frac{\partial e}{\partial r}-\frac{\gamma_{e} \theta_{o}}{\rho C_{1}^{2}}\left(1+\gamma_{o} \frac{\partial}{\partial t}\right) \frac{\partial \theta}{\partial r}$

$\nabla^{2} \theta+\frac{K^{*} C_{1}^{2} \eta}{K} \nabla^{2} \dot{\theta}=\eta C_{1}^{2} \ddot{\theta}+\frac{\gamma_{e} C_{1}^{2}}{K}\left(1+\gamma_{o} \frac{\partial}{\partial t}\right) \ddot{e}$

where, $\eta=\frac{\rho C_{E}}{K}, C_{1}^{2}=\frac{\lambda_{e}+2 \mu_{e}}{\rho}$

Equation (23) can be written in the form:

$e=\left(1+\mu_{o} H_{o}^{2}+\frac{\lambda_{e} \alpha_{o}+2 \mu_{e} \alpha_{1}}{\rho C_{1}^{2}} \frac{\partial}{\partial t}\right) \nabla^{2} e-\frac{\gamma_{e} \theta_{o}}{\rho C_{1}^{2}}\left(1+\gamma_{o} \frac{\partial}{\partial t}\right) \nabla^{2} \theta$

\section{LAPLACE TRANSFORM DOMAIN}

Taking the Laplace transform of equations (23)-(31) by using homogeneous initial conditions, defined and denoted as

$\bar{f}(s) \int_{0}^{\infty} e^{-s t} f(t) d t \quad, s>0$

We obtain

$\bar{J}=\frac{d \bar{e}}{d r}$

$\bar{h}=-\left(\frac{d \bar{u}}{d r}+\frac{\bar{u}}{r}\right)$

$\bar{E}=s \bar{u}$

$\nabla^{2} \bar{\theta}=L_{1} \bar{\theta}+L_{1} \bar{e}$

(35)

$\bar{\sigma}_{r r}=a_{1} \frac{d \bar{u}}{d r}+a_{2} \bar{e}-a_{3} \bar{\theta}$,

(36)

$\bar{\sigma}_{\phi \phi}=\bar{\sigma}_{\theta \theta}=a_{1} \frac{\bar{u}}{r}+a_{2} \bar{e}-a_{3} \bar{\theta}$

(37)

$\nabla^{2} \bar{e}=M_{1} \bar{\theta}+M_{1} \bar{e}$

(38)

where

$L_{1}=\frac{K \eta C_{1}^{2} s^{2}}{K+K^{*} \eta C_{1}^{2} s^{2}}, l_{2}=\frac{\gamma_{e} C_{1}^{2}\left(1+\gamma_{o} s\right) s^{2}}{K+K^{*} \eta C_{1}^{2} s^{2}}$

$, a_{1}=\frac{2 \mu_{e}}{\lambda_{e}+2 \mu_{e}}\left(1+\alpha_{1} s\right), a_{2}=\frac{\lambda_{e}}{\lambda_{e}+2 \mu_{e}}\left(1+\alpha_{o} s\right)$

$a_{3}=\frac{\gamma_{e} \theta_{o}}{\lambda_{e}+2 \mu_{e}}\left(1+\gamma_{o} s\right), M_{1}=\frac{\gamma_{e} \theta_{o}\left(1+\gamma_{o} s\right) L_{1}}{\mu_{o} H_{o}^{2} \rho C_{1}^{2}+\rho C_{1}^{2}+\left(\lambda_{e} \alpha_{o}+2 \mu_{e} \alpha_{1}\right) s}$

,$M_{2}=\frac{\rho C_{1}^{2} s^{2}+\gamma_{e} \theta_{o}\left(1+\gamma_{o} s\right) L_{2}}{\mu_{o} H_{o}^{2} \rho C_{1}^{2}+\rho C_{1}^{2}+\left(\lambda_{e} \alpha_{o}+2 \mu_{e} \alpha_{1}\right) s}$

\section{STATE SPACE FORMULATION}

Choosing as state variables the temperature of heat conduction $\bar{\theta}$ and the strain components $\bar{e}$ then equations (35) and (38) can be written in the matrix form

$\nabla^{2} V(r, s)=A(s) \bar{V}(r, s)$

where

$\bar{V}(r, s)=\left[\begin{array}{l}\bar{\theta}(r, s) \\ \bar{e}(r, s)\end{array}\right], A(s)=\left[\begin{array}{ll}L_{1} & L_{2} \\ M_{1} & M_{2}\end{array}\right]$

The formal solution of system (39) can be written in the form

$\bar{V}(r, s)=C \frac{e^{-\sqrt{A(s)} r}}{r}+B \frac{e^{-\sqrt{A(s)} r}}{r}$

For bounded solution with large $r$, we have canceled the exponential part has positive power. And at $r=R$ the value of $C$ is given by

$C=R \bar{V}(R, s) e^{\sqrt{A(s)} R}$, then equation (40) reduces to

$\bar{V}(r, s)=\frac{R}{r} \bar{V}(R, s) e^{-\sqrt{A(s)} R} \quad, r \geq R$

We will use the will-known Cayley-Hamiltonian theorem to find the form of the matrix

$\exp (-\sqrt{A(s)}(r-R))$

The characteristic equation of the matrix $A(s)$ can be written as $k^{2}-\left(L_{1}+M_{2}\right) k+\left(L_{1} M_{2}-L_{2} M_{1}\right)$

The roots of this equation namely $k_{1}$ and $k_{2}$ satisfy the relations $k_{1}+k_{2}=L_{1}+M_{2}$ 
$k_{1} k_{2}=L_{1} M_{2}-L_{2} M_{1}$

The Tailor's series expansion for the matrix exponential of

$\exp (-\sqrt{A(s)}(r-R))$ is given by

$\exp (-\sqrt{A(s)}(r-R))=\sum_{n=0}^{\infty} \frac{[-\sqrt{A(s)}(r-R)]^{n}}{n !}$

Using Cayley - Hamiltonian theorem, we can express $A^{2}$ and

higher orders of the matrix $A$ in terms of $I$ and $A$

where $I$ is the unit matrix of second order.

Thus, the infinite series in equation (42) can be reduced to

$\exp (-\sqrt{A(s)}(r-R))=b_{o}(r, s) I+b_{1}(r, s) A$

Where $b_{o}$ and $b_{1}$ are some coefficients depending on $s$ and $r$.

By Cayley- Hamiltonian theorem, the characteristic roots $k_{1}$ and

$k_{2}$ of the matrix $A$ must satisfy equation (42), thus we have

$\exp \left(-\sqrt{k_{1}}(r-R)\right)=b_{o}+b_{1} k_{1}$

$\exp \left(-\sqrt{k_{2}}(r-R)\right)=b_{o}+b_{1} k_{2}$

Solving the above linear system of equations, we get

$b_{o}=\frac{k_{1} e^{-\sqrt{k_{2}}(r-R)}-k_{2} e^{-\sqrt{k_{1}}(r-R)}}{k_{1}-k_{2}}$

$b_{1}=\frac{e^{-\sqrt{k_{1}}(r-R)}-e^{-\sqrt{k_{2}}(r-R)}}{k_{1}-k_{2}}$

Hence, we have

$\exp (-\sqrt{A(s)}(r-R))=L_{i j}(r, s), \quad i, j=1,2$

where,

$L_{11}=\frac{e^{-\sqrt{k_{2}}(r-R)}\left(k_{1}-L_{1}\right)+e^{-\sqrt{k_{1}}(r-R)}\left(L_{1}-k_{2}\right)}{k_{1}-k_{2}}$

$L_{12}=\frac{L_{2} e^{-\sqrt{k_{1}}(r-R)}-L_{1} e^{-\sqrt{k_{2}}(r-R)}}{k_{1}-k_{2}}$

$L_{21}=\frac{M_{1} e^{-\sqrt{k_{1}}(r-R)}-M_{2} e^{-\sqrt{k_{1}}(r-R)}}{k_{1}-k_{2}}$

$L_{22}=\frac{e^{-\sqrt{k_{1}}(r-R)}\left(M_{2}-k_{2}\right)+e^{-\sqrt{k_{2}}(r-R)}\left(k_{1}-M_{2}\right)}{k_{1}-k_{2}}$

\section{APPLICATIONS}

In order to evaluate the unknown parameters $\bar{\theta}_{o}(r, s)$ and $\bar{e}_{o}(r, s)$, we shall use the boundary conditions on the internal surface of the shell, $r=$ $R$ which are given by:

(I) Thermal boundary condition at $r=R$

$\theta(R, t)=\theta_{o}$

Taking the Laplace transform, this is defined as following:

$\bar{\theta}_{o}(r, s)=\frac{\theta_{o}}{s}$

\section{(II) Mechanical boundary condition}

The internal surface $r=R$ has a rigid foundation, which is rigid enough to prevent any strain

$e(R, t)=0$

Taking the Laplace transform, this is defined as following:

$\bar{e}(R, s)=\bar{e}_{o}=0$

Using the conditions (49) and (50) into equation (34) and using equations (44)-(48), we get

$$
\begin{aligned}
& \bar{\theta}(r, s)=\frac{R \theta_{o}}{s\left(k_{1}-k_{2}\right) r}\left[\left(k_{1}-L_{2}\right) e^{-\sqrt{k_{2}}(r-R)}+\left(k_{2}-L_{1}\right) e^{-\sqrt{k_{2}}(r-R)}\right] \\
& \bar{e}(r, s)=\frac{R M_{1} \theta_{o}}{s\left(k_{1}-k_{2}\right) r}\left[\left(k_{2}-L_{1}\right) e^{-\sqrt{k_{1}}(r-R)}+\left(k_{1}-L_{2}\right) e^{-\sqrt{k_{2}}(r-R)}\right]
\end{aligned}
$$

To find the displacement, taking Laplace transform for equation (23) and using equations (51) and (52), we get $\bar{u}(r, s)=\frac{R \theta_{o}}{s r^{2}\left(k_{1}-k_{2}\right) r}\left(\left(\left(1+r \sqrt{k_{1}}\right)\left(B_{5}\left(L_{1}-k_{2}\right)\right)-\right.\right.$

$\left.B_{4} M_{1}\right) e^{-\sqrt{k_{1}}(r-R)}+\left(\left(1+r \sqrt{k_{2}}\right)\left(B_{4} M_{1}+B_{5}\left(k_{1}-\right.\right.\right.$

$\left.\left.\left.\left.L_{1}\right)\right)\right) e^{-\sqrt{k_{2}}(r-R)}\right)$

To find the stresses, from equations (36), (37) and (58)- (60) we get

$\bar{\sigma}_{r r}=\frac{R \theta_{o}}{s r^{2}\left(k_{1}-k_{2}\right) r}\left\{e^{-\sqrt{k_{1}}(r-R)}\left(M_{1}\left(B_{1}+B_{3}\right) r^{2}+2 B_{1} B_{4} M_{1}(1+\right.\right.$

$\left.\left.\left.r \sqrt{k_{1}}\right)-\left(L_{1}-k_{2}\right)\left(B_{3} r^{2}+2 B_{1} B_{5}\left(1+r \sqrt{k_{1}}\right)\right)\right)+e^{-\sqrt{k_{2}}(r-R)}\right\}$

where,

$B_{4}=\frac{1}{s^{2}}+\frac{\lambda_{e} \alpha_{o}+2 \mu_{e} \alpha_{1}}{\rho C_{1}^{2} s^{2}}$

$B_{5}=\frac{\gamma_{e} \theta_{o}\left(1+\gamma_{o} s\right)}{\rho C_{1}^{2} s^{2}}$

\section{NUMERICAL INVERSION OF LAPLACE TRANSFORMS}

In order to invert the Laplace transforms in the above equations we shall use a numerical technique based on Fourier expansions of functions. Let $\bar{g}(s)$ be the Laplace transform of a given function $g(t)$. The inversion formula of Laplace transforms states that

$g(t)=\frac{1}{2 \pi i} \int_{d-i \infty}^{d+i \infty} e^{s t} \bar{g}(s) d s$

Where $d$ is an arbitrary positive constant greater than all the real parts of the singularities of $\bar{g}(s)$. Takings $=d+i y$, we get

$g(t)=\frac{e^{d t}}{2 \pi} \int_{-\infty}^{\infty} e^{i k y} \bar{g}(d+i y) d y$

This integral can be approximated by

$g(t)=\frac{e^{d t}}{2 \pi} \sum_{k=-\infty}^{\infty} e^{i k t \Delta y} \bar{g}(d+i k \Delta y) \Delta y$

Taking $\Delta y=\frac{\pi}{t_{1}}$ we obtain:

$g(t)=\frac{e^{d t}}{t_{1}}\left(\frac{1}{2} \bar{g}(d)+\operatorname{Re}\left(\sum_{k=1}^{\infty} e^{i k t \pi / t_{1}} \bar{g}\left(d+i k \pi / t_{1}\right)\right)\right)$

For numerical purposes this is approximated by the function

$g_{N}(t)=\frac{e^{d t}}{t_{1}}\left(\frac{1}{2} \bar{g}(d)+\operatorname{Re}\left(\sum_{k=1}^{N} e^{i k t \pi / t_{1}} \bar{g}\left(d+i k \pi / t_{1}\right)\right)\right)$

where $N$ is a sufficiently large integer chosen such that

$\frac{e^{d t}}{t_{1}} \operatorname{Re}\left(e^{i N \pi t / t_{1}} \bar{g}\left(d+i N \pi / t_{1}\right)\right)<\eta$

Where $\eta$ is a reselected small positive number that corresponds to the degree of accuracy to be achieved Formula (49) is the numerical inversion formula valid for $0 \leq t \leq t_{1}$. In particular, we chooset $=t_{1}$, getting

$g_{N}(t)=\frac{e^{d t}}{t_{1}}\left(\frac{1}{2} \bar{g}(d)+\operatorname{Re}\left(\sum_{k=1}^{N}(-1)^{k} \bar{g}\left(d+i k \pi / t_{1}\right)\right)\right)$

\section{NUMERICAL RESULTS AND DISCUSSIONS}

The copper material was chosen for purposes of numerical evaluations and constants of the problem were taken as following 
$\mu_{e}=3.86 \times 10^{10} \mathrm{~N} / \mathrm{m}^{2}, \lambda_{e}=7,76 \times 10^{10} \mathrm{~N} / \mathrm{m}^{2}, \alpha_{o}=$ $6.8831 \times 10^{-13}, \alpha_{o}=6.8831 \times 10^{-13}, R=1, \theta_{o}=1$

$K=386 \mathrm{~N} / \mathrm{Ks}, \alpha_{t}=17.8 \times 10^{-5} \mathrm{~K}^{-1}, C_{E}=383.1 \mathrm{~m}^{2} / \mathrm{K}$, $T_{o}=293 \mathrm{~K}, \rho=8954 \mathrm{~kg} / \mathrm{m}^{2}$

In order to study the effect of time $t$ on temperature, radial stress, shear stress, displacement and strain, we now present our results in the form of graphs (Figs. 1-5). Fig. 1 shows the variation of temperature $\theta$ against $r$ for wide range ofr, $1 \leq \mathrm{r} \leq 3$ for different values of time ( $t=0.5, t=0.05$ and $t=0.005)$ and we have noticed that, the time $t$ has significant affects on temperature. The increasing of the value of $t$ causes decreasing of the value of temperature, and temperature $\theta$ vanishes more rapidly.

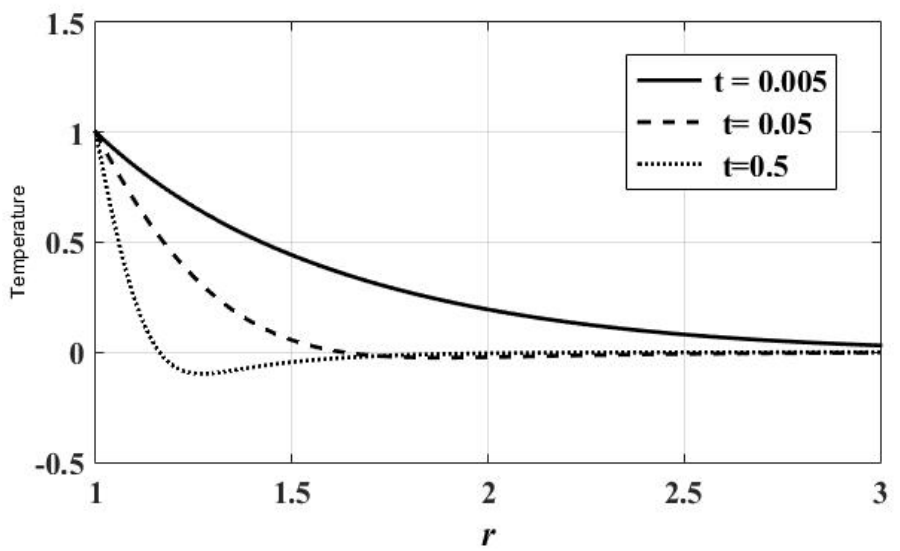

Fig. 1 Variation of Temperature $\theta$ with distance $r$

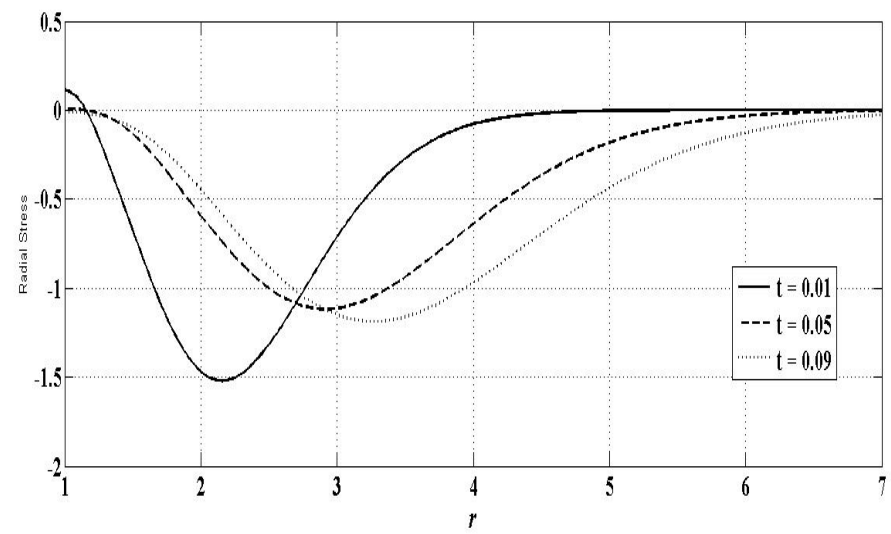

Fig. 2 Variation of Radial Stress $\sigma_{\text {rrwith distance } r}$

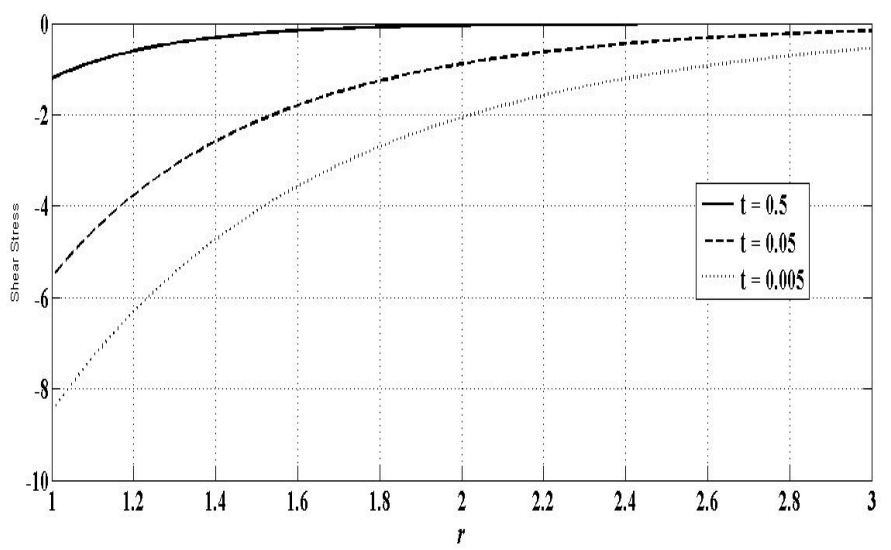

Fig. 3 Variation of Shear Stress $\sigma_{\varphi \varphi}$ with distance $r$

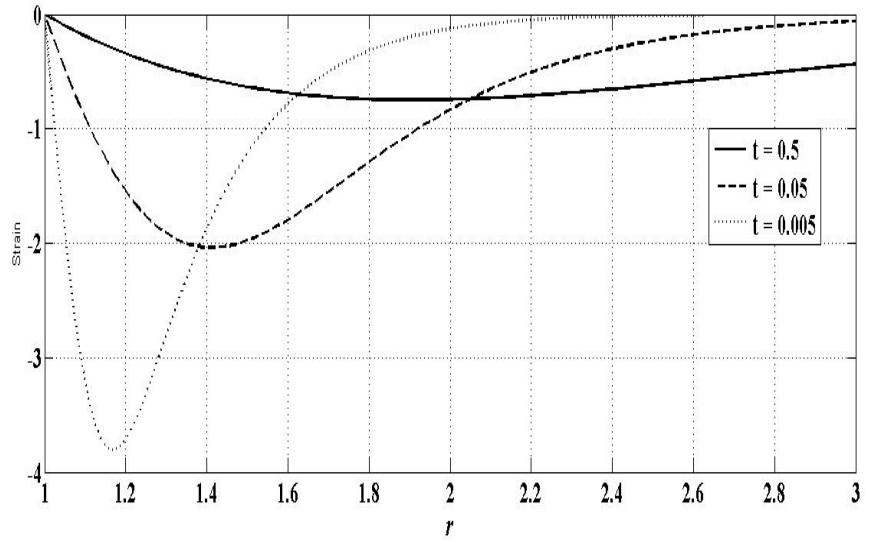

Fig. 4 Variation of Strain e with distance $r$

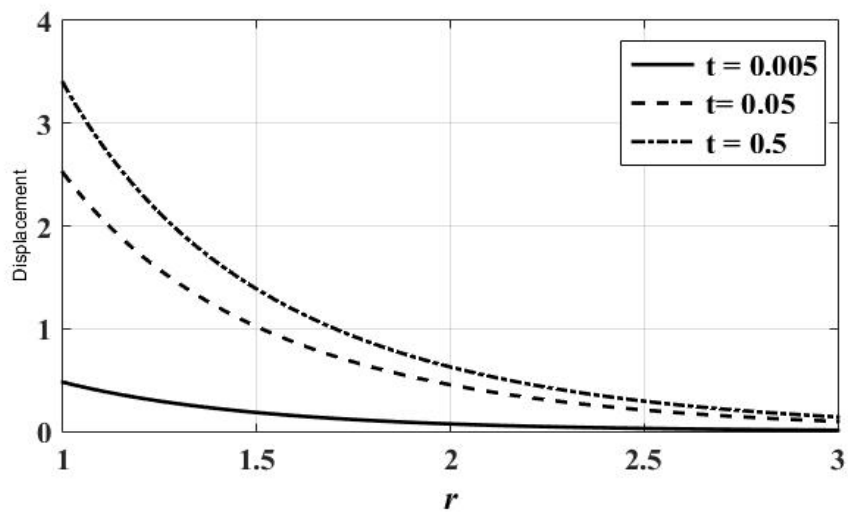

Fig. 5 Variation of Displacement $u$ with distance $r$

Fig. 2 shows the variation of the radial stress $\sigma_{r r}$ against $r$ for wide range of $r,(1 \leq r \leq 7)$ for different values of time $(t=0.01, t=$ $0.05, t=0.09)$ and we have noticed that, the time $t$ has significant effects on stress, for wide range of $r(1 \leq r \leq 2.7)$ the increasing of the value of $t$ causes decreasing of the value of the radial stresses $\sigma_{r r}$ and for wide range of $r(2.7 \leq r \leq 8)$ the increasing of the value of $t$ causes increasing of the value of the radial stresses and radial stresses $\sigma_{r r}$ vanishes more rapidly.

Fig. 3 shows the variation of the shear stress $\sigma_{r r}$ against $r$ for wide range of $r(1 \leq \mathrm{r} \leq 3)$, for different values of time $(t=0.5, t=$ $0.05, t=0.005)$ and we have noticed that, the time $t$ has significant effects on stress. The increasing of the value of $t$ causes decreasing of the value of the shear stress, and the shear stress $\sigma_{r r}$ vanishes more rapidly.

Fig. 4 shows the variation of strain $e$ against $r$ for wide range of $r, 1 \leq r \leq 3$, for different values of time $(t=0.5, t=0.05, t=$ $0.005)$ and we have noticed that, the time $t$ has significant effects on strain, for the wide range $(1 \leq r \leq 1.4)$ the increasing of the value of $t$ causes decreasing of the value of strain, for the wide range $(1.4 \leq r \leq$ 3 ) the increasing of the value of $t$ causes increasing of the value of strain and strain $\mathrm{e}$ vanishes more rapidly.

Fig. 5 shows the variation of displacement $u$ against $r$ for wide range of $r(1 \leq \mathrm{r} \leq 3)$, for different values of time $(t=0.5, t=0.05, t=$ $0.005)$ and we have noticed that, the time $t$ has significant effects on displacement $u$. The increasing of the value of $t$ causes increasing of the value of displacement $u$, and displacement $u$ vanishes more rapidly. 


\section{CONCLUSIONS}

According to this work, many researchers in the field of generalized thermo elasticity have applied Green- Naghdi theory (III-II) for thermo elastic problem and very few of them can successfully applied for magneto thermo Viscoelastic problem. In this paper we deduce that the magnitude of the all physical quantities depend on time

$t$.It can be noted that the speed of propagation of all physical quantities is finite and coincide with the physical behavior of Viscoelastic material.

\section{ACKNOWLEDGEMENTS}

The authors wish to acknowledge the approval and the support of this research study by the Project NO. 7502 -SCI - 2017 - 1 - 8-F from the Deanship of Scientific Research in Northern Border University, Arar, KSA.

\section{Nomenclature}

$\begin{array}{ll}\lambda_{e}, \mu_{e} & \text { Lame elastic constants } \\ \rho & \text { Density } \\ C_{E} & \text { Specific heat at constant strain } \\ K & \text { Thermal conductivity } \\ \alpha_{t} & \text { Coefficient of linear thermal expansion } \\ \gamma_{e} & \left(3 \lambda_{e}+2 \mu_{e}\right) \alpha_{t} \\ \gamma_{o} & \left(3 \lambda_{e} \alpha_{o}+2 \mu_{e} \alpha_{1}\right) \alpha_{t} / \gamma_{e} \\ \alpha_{o}, \alpha_{1} & \text { Viscoelastic relaxation time } \\ t & \text { Time } \\ q_{i} & \text { Components of heat flux vector } \\ \sigma_{i j} & \text { Components of stress tensor } \\ e_{i j} & \text { Components of strain tensor } \\ u_{i} & \text { Components of displacement vector } \\ T_{o} & \text { Reference temperature } \\ \theta & \text { Temperature increment } \\ \delta_{i j} & \text { Kronicker delta } \\ e & \text { Cubical dilatation } \\ R & \text { Radius of the shell } \\ \mu_{o} & \text { Magnetic permittivity } \\ \boldsymbol{E} & \text { Electric displacement vector } \\ \boldsymbol{J} & \text { Current density vector } \\ \boldsymbol{H} & \text { Total magnetic intensity vector } \\ \boldsymbol{h} & \text { Induced magnetic field vector } \\ \boldsymbol{H}_{\boldsymbol{o}} & \text { Initial uniform magnetic field } \\ \boldsymbol{F}_{\boldsymbol{i}} & \text { Components of Lorentz body force } \\ & \end{array}$

\section{REFERENCES}

Bagley, R.L., and Torvik, P.J., 1983, "A different approach to the analysis of viscoelastically damped structure," AIAA. J, 21, $741-748$. http://dx.doi.org/10.2514/3.8142

Bahar, L., and Hetnarski, R., 1978, "State Space Approach to Thermo elasticity," J. Thermal Stresses, 1, 135 - 145. http://dx.doi.org/10.1080/01495737808926936

Biot, M., 1956, "Thermo elasticity and Irreversible Thermo-Dynamics," J. Appl. Phys, 27, $240-253$. http://dx.doi.org/10.1063/1.1722351

Dhaliwal, S., and Hany, H., 1980, "Generalized Thermo elasticity for Anisotropic Media," Quart. Appl. Math, 303, 1-8.

http://dx.doi.org/10.1090/qam/575828
Duhamel, J.H., 1938, "Second mémoire sur les phénomènes thermomécanique," J. de L'École Polytechnique.

El-Bary, A.A., and Atef, M., 2016, "Modified Approach for Stress Strain Equation in the Linear Kelvin-Voigt Solid Based on Fractional Order," j. Comput. Theor. Nanosci, 13(1), 579-583.

http://dx.doi.org/10.1166/jctn.2016.4843

El-Karamany, A.S., Ezzat, M.A., and El-Bary, A.A., 2018, “Thermo diffusion with two time delays and Kernel functions," Mathematics and Mechanics of Solids, 23 (2), 195-208.

http://dx.doi.org/10.1177/1081286516676870

Ezzat, M.A., and El-Bary, A.A., 2017, "On thermo-viscoelastic infinitely long hollow cylinder with variable thermal conductivity," Microsyst Technol , 23 (8), 3263-3270. http://dx.doi.org/10.1007/s00542-016-3101-2

Ezzat, M.A., and El-Bary, A.A., 2009, "On three models of magnetohydrodynamic free- convection flow," Canadian. J. Phy, 87,1213-1226. http://dx.doi.org/10.1139/P09-071

Ezzat, M.A., and El-Bary, A.A., 2012, "MHD free convection flow with fractional heat conduction law," MAGNETOHYDRODYNAMICS, 48(4), 503-522.

http://dx.doi.org/10.22364/mhd.48.4.1

Ezzat, M.A., and El-Bary, A.A., 2014, "Two-temperature theory of magneto-thermo-viscoelasticity with fractional derivative and integral orders heat transfer," Journal of Electromagnetic Waves and Applications, 28(16).

http://dx.doi.org/10.1080/09205071.2014.953639

Ezzat, M.A., and El-Bary, A.A., 2015, "Memory-dependent derivatives theory of thermo-viscoelasticity involving two-temperature," Journal of Mechanical Science and Technology, 29 (10), 4273-4279. http://dx.doi.org/10.1007/s12206-015-0924-1

Ezzat, M.A., and El-Bary, A.A., 2015, "State space approach to twodimensional magneto-thermoelasticity with fractional order heat transfer in a medium of perfect conductivity," International Journal of Applied Electromagnetics and Mechanics, 49, 607-625. http://dx.doi.org/10.3233/JAE-150095

Ezzat, M.A., and El-Bary, A.A., 2017, "Fractional magnetothermoelastic materials with phase-lag Green-Naghdi theories," STEEL AND COMPOSITE STRUCTURES, 24 (3), 297-307. http://dx.doi.org/10.1007/s00542-017-3425-6

Ezzat, M.A., and El-Bary, A.A., 2018, "Electro-magneto interaction in fractional green-Naghdi thermoelastic solid with a cylindrical cavity," J. Waves in Random and Complex Media, 28(1), 150-168. http://dx.doi.org/10.1080/17455030.2017.1332798

Ezzat, M.A., and El-Bary, A.A., 2018, "Thermoelectric spherical shell with fractional order heat transfer," Microsystem Technologies, 24 (2), 891-899.

http://dx.doi.org/10.1007/s00542-017-3400-2

Ezzat, M.A., El Karamany, A.S., and El-Bary, A.A., 2017, "Thermoelectric viscoelastic materials with memory-dependent derivative," Smart Structures and Systems, 19(5) 539-551.

http://dx.doi.org/10.12989/sss.2017.19.5.539 
Ezzat, M.A., El Karamany, A.S., El-Bary, A.A., 2016, "Electrothermoelasticity theory with memory-dependent derivative heat transfer," International Journal of Engineering Science, 99, 22-38. http://dx.doi.org/10.1016/j.ijengsci.2015.10.011

Ezzat, M.A., El Bary, A. A., 2016, "Effects of variable thermal conductivity on Stokes' flow of a thermoelectric fluid with fractional order of heat transfer," International Journal of Thermal Sciences, 100, 305-315.

http://dx.doi.org/10.1016/j.ijthermalsci.2015.10.008

Ezzat, M.A., El-Bary, A. A., 2016, "Modeling of fractional magnetothermoelasticity for a perfect conducting materials," SMART STRUCTURES AND SYSTEMS, 18(4), 707-731.

http://dx.doi.org/10.12989/sss.2016.18.4.707

Ezzat, M.A., El-Bary, A.A., El- Karamany, A.S., 2009, "Tow temperature theory in generalized magneto thermo viscoelasticity," Canadian. J. Phy, 87, 329-336.

http://dx.doi.org/10.1139/P08-143

Ezzat, M.A., El-Karamany, A.S., and El-Bary, A.A., 2015, “On Thermoviscoelasticity with Variable Thermal Conductivity and Fractional-Order Heat Transfer," International Journal of Thermophysics ,36 (7), 16841697.

http://dx.doi.org/10.1007/s10765-015-1873-8

Ezzat, M.A., Zakaria, M., and El-Bary, A.A., 2010, "Thermo-ElectricVisco-Elastic Material," Journal of applied Polymer Science, 117, 19341944.

http://dx.doi.org/10.1002/app.32170

Ezzat, M.A.,El-Karamany, A.S., and El-Bary, A.A.,2015, "Thermoviscoelastic materials with fractional relaxation operators," Applied Mathematical Modeling, 39(23), 7499-7512.

http://dx.doi.org/10.1016/j.apm.2015.03.018

Green, A.E., and Lindsay, K.A., “Thermo elasticity,”, J. Elasticity, 2, 1 -7 .

http://dx.doi.org/10.1007/BF00045689

Hany, H., 1980, "On Generalized Thermo elasticity,” Ph. D. Thesis, University of Calgary Canada.

Hetnarski, R., 1961, “Coupled One-dimensional Thermal Shock problem for Small Times,” Arc. Mech, 13, 295 - 306.

Hetnarski, R., 1964, "The Fundamental Solution of the Couple Thermo elastic Problem for Small Times," Arch, Mech, Stos, 16, 23 - 32.

Ignaczak, J., 1959 “, Dynamic Thermo elastic Problem of a Spherical Cavity”, Arch, Mech. Stos, 11, $399-408$.

Ismail, M.A.H., Khamis, A.K., El-Bary, A.A. , and. Youssef, H.M, 2017, "Effect of rotation of generalized thermoelastic layer subjected to harmonic heat : state - space approach," Microsystem Technologies , 23 (8), 3381-3388.

http://dx.doi.org/10.1007/s00542-016-3137-3
Khamis, A.K., Ismail, A.H., Youssef, H.M., and El-Bary, A.A., 2017, "Thermal shock problem of two-temperature generalized thermoelasticity without energy dissipation with rotation," Microsystem Technologies, 23 (10), 4831-4839. http://dx.doi.org/10.1007/s00542-017-3279-y

Koltunov, M.A., 1976, “Creeping and Relaxation,” Moscow.

Lord, H. and Shulman, Y., 1976, "A Generalized Dynamical Theory of Thermo elasticity," J. Mech. Phys. Solid., 15, 299 - 309. http://dx.doi.org/10.1016/0022-5096(67)90024-5

Mishra, J.C., Chakrabarty, A.K., Samanta, S.C., and Mishra, S.C., 1992, "Thermally induced vibration in an infinite anisotropic viscoelastic solid with a cylindrical cavity in the presence of a uniform axial magnetic field," Int. J. Eng. Sci, 31 , $1047-1052$. http://dx.doi.org/10.1016/0020-7225(93)90112-8

Neumann, F.,1841,"Vorlesungen Uber dies Theorie der Elasticitat," Meyer\& Brestau.

Nickell and Sackman, J., 1968, "Variational Principles for Linear Coupled Thermo elasticity," Quart. Math, 26, $11-26$.

Nowacki, W., 1957, “A Dynamical Problem of Thermo elasticity,” Arch. Mech. Stos, 9,325 - 334.

Paria, G., 1962, "On magneto-thermo-elastic plane waves," Proc. Cambridge Phil. Soc, 58, 527 - 531.

Paria, G., 1976, "Magneto-elasticity and Magneto-thermo-elasticity," Adv. Appl. Mech, 10, $73-112$.

Takeuti, Y., and Tsuji, M., 1982, "Transient Thermal Stresses in a Plate due to Rolling with Consideration of Coupling Effect," J. Thermal Stresses, 5, $53-65$.

Weiner, J., 1957, “A Uniqueness Theorem for Coupled Thermo elastic Problem. Quart,” Appl. Math, 15, 102 - 105.

Youssef, M. and El-Bary, A.A., 2018, "The reference temperature dependence of Young's modulus of two-temperature thermoelastic damping of gold Nano-beam," Mechanics of Time-Dependent Materials, 22(4), 435-445.

http://dx.doi.org/10.1007/s11043-017-9365-9

Youssef, M., and El-Bary, A.A., 2014, "Thermoelastic Material Response due to Laser Pulse Heating in Context of Four Theorems of Thermoelasticity," J. Thermal Stresses, 37, 1379-1389. http://dx.doi.org/10.1080/01495739.2014.937233

Youssef, M., El-Bary, A.A., and Elsibai, A., 2014, “”, Vibration of Gold Nano Beam in Context of Two-Temperature, Generalized Thermoelasticity Subjected to Laser Pulse, Latin American Journal of Solids and Structures, 11, 2460-2482. http://dx.doi.org/10.1590/S1679-78252014001300008

Youssef, M., Elsibai, A., and El-Bary, A.A., 2017, "Effect of the speed, the rotation and the magnetic field on the Q-factor of an axially clamped gold micro-beam," Meccanica, 52 (7), 1685-1694.

http://dx.doi.org/10.1007/s11012-016-0498-8 